\title{
THE LATTICE OF CLOSED CONGRUENCES ON A TOPOLOGICAL LATTICE
}

BY

\author{
DENNIS J. CLINKENBEARD
}

\begin{abstract}
Our primary objectives are:
(1) if $L$ is a lattice endowed with a topology making both the meet and join continuous then

(i) the natural map which associates a congruence with the smallest topologically closed congruence containing it preserves finite meets and arbitrary joins;

(ii) the lattice of such closed congruences is a complete Brouwerian lattice;

(2) if $L$ is a topological (semi) lattice with the unit interval as a (semi) lattice homomorphic image then the lattice of closed (semi) lattice congruences has no compatible Hausdorff topology.
\end{abstract}

Introduction. Given a topological lattice $L$, we investigate $\theta^{*}(L)$ the lattice of all congruences on $L$ which are topologically closed in $L \times L$. This collection of congruences is particularly interesting when $L$ is compact Hausdorff since each member of $\theta^{*}(L)$ will then preserve many algebraic and topological properties of $L$.

It is well known that for any lattice $L$ its congruence lattice $\theta(L)$ is both algebraic and satisfies the infinite distributive equation $\left(\bigvee_{\alpha \in \mathscr{Q}} x_{\alpha}\right) \wedge y=$ $\bigvee_{\alpha \in \mathscr{Q}}\left(x_{\alpha} \wedge y\right)^{2}$ which characterizes complete Brouwerian lattices (cf. [1, pp. 188, $138])$. Yet the only known result for $\theta^{*}(L)$ similar to the defining equation for Brouwerian lattices is by A. R. Stralka, who proved that if $L$ is a compact Hausdorff distributive topological lattice of finite breadth, then $\theta^{*}(L)$ is a distributive lattice (cf. [6]).

In $\S 1$ we demonstrate that $\theta(L)$ and $\theta^{*}(L)$ are much more similar than the above result suggests. By merely assuming $L$ has a topology (not necessarily Hausdorff) such that both the meet and join are continuous we prove that $\theta^{*}(L)$ is also a complete Brouwerian lattice. Furthermore, the preservation properties of the natural map between $\theta(L)$ and $\theta^{*}(L)$ mentioned in the abstract allows us to obtain another fact: $L$ is Hausdorff iff the pseudo-complements of $\theta(L)$ and $\theta^{*}(L)$ are identical. From this we conclude immediately that $L$ is compact Hausdorff iff all pseudo-complements in $\theta(L)$ have compact Hausdorff images.

As mentoned previously $\theta(L)$ is also algebraic. In general, the congruence lattice

Received by the editors June 27, 1979.

AMS (MOS) subject classifications (1970). Primary 06A22; Secondary 06A23.

Key words and phrases. Brouwerian lattice, congruence lattice, compact Hausdorff topological lattice.

${ }^{1}$ Major portions of this paper were prepared from a dissertation completed under A. R. Stralka at the University of California at Riverside.

${ }^{2}$ Throughout this paper $\mathscr{Q}$ will always be some arbitrary index set. 
on any partial algebra is algebraic. Thus, $\theta(L)$ is algebraic when $L$ is also a semilattice. Moreover, any algebraic lattice admits a compact Hausdorff (zero-dimensional) order-compatible topology ${ }^{3}$ (cf. [5]). In contrast, the only known analogue for $\theta^{*}(L)$ was proven by K. H. Hofmann and M. W. Mislove in [4]. The result can be stated as follows: if $L$ is an algebraic lattice such that all its quotients are also algebraic when only maps preserving arbitrary meets and directed joins are considered $^{4}$ then the opposite lattice of $\theta^{*}(L)$ is an algebraic lattice; hence, in particular, $\theta^{*}(L)$ has a compatible compact Hausdorff (zero-dimensional) topology. In $\$ 2$ we prove that whenever $L$ is either a topological lattice or topological semilattice then $\theta^{*}(L)$ has no compatible Hausdorff topology if $L$ has the unit interval as a homomorphic image. Hence, topologically $\theta(L)$ and $\theta^{*}(L)$ are, in general, quite different.

In the Appendix we exhibit some examples which expand on the material in $\$ 1$.

Preliminaries. Given a lattice $L$, let $D=\{(x, x) \mid x \in L\}$. A subset $X$ of $L \times L$ is said to be a $D$-ideal iff $D \cup(D \vee X) \cup(D \wedge X) \subseteq X$. A lattice congruence (or merely congruence) $[\phi]$ is an equivalence relation on $L$ which is also a $D$-ideal of $L \times L$.

Note that for any lattice $L, D$ and $L \times L$ are congruences.

For each lattice surmorphism $\phi$ from $L$ onto a lattice $M$, there is a unique congruence $[\phi]=\{(x, y) \mid \phi(x)=\phi(y)\}$. Conversely, for each congruence $[\phi]$ we obtain both a unique image of $L, \phi(L)=\{\bar{x} \subseteq L \mid y \in \bar{x}$ iff $(x, y) \in[\phi]\}$, and a unique lattice homomorphism $\phi$ from $L$ onto $\phi(L)$ such that $\phi(x)=\bar{x}$.

By a topological lattice $L$ we mean that $L$ is a lattice endowed with a topology such that both the meet and join are continuous binary functions from $L \times L$ into $L$.

Given a topological lattice $L$, we assume that for any $[\phi] \in \theta(L), \phi(L)$ has the inherited quotient topology; thus, $\phi$ becomes a continuous homomorphism. ${ }^{5}$

If $X$ is some predetermined topological space and $Y \subseteq X$ then $\mathrm{Cl} Y$ will denote the closure of $Y$ in $X$.

If $[\phi]=\mathrm{Cl}[\phi]$ in $L \times L$, then $[\phi]$ is called a closed congruence. The complete lattice $\theta^{*}(L)$ of all closed congruences has the same meet as $\theta(L)$, namely set-theoretic intersection. Thus, we use the usual intersection symbol " $\cap$ " to represent both meets. However, we distinguish between the joins of $\theta(L)$ and $\theta^{*}(L)$ by letting " $\bigvee$ " denote the join in $\theta(L)$ and " $\forall$ " the join in $\theta^{*}(L)$. In general, all that can be said regarding the joins is that if $\left\{\left[\phi_{\alpha}\right] \mid \alpha \in \mathbb{Q}\right\} \subseteq \theta^{*}(L)$ then $\bigvee_{\alpha \in \mathcal{Q}}\left[\phi_{\alpha}\right]$ $\subseteq \forall_{\alpha \in \mathcal{Q}}\left[\phi_{\alpha}\right]$.

The primary reasons for examining closed congruences occur when $L$ is a compact Hausdorff topological lattice. First, it is well known from general topology that for any compact Hausdorff topological space $X$ and any equivalence relation

\footnotetext{
${ }^{3}$ By compatible we mean that order convergence of chains implies topological convergence.

${ }^{4}$ In other words, $L$ and all of its compact semilattice quotients are zero dimensional.

${ }^{5}$ It is not difficult to show that $\phi(L)$ is a topological lattice. But the fact that the composition of the two continuous functions, meet (respectively, join) in $L$ and $\phi$, produces a continuous function does not suffice to prove that the meet (respectively, join) in $\phi(L)$ is continuous.
} 
$E$ on $X, E$ is closed in $X \times X$ iff the quotient space $X / E$ is Hausdorff. Thus, whenever $L$ is compact Hausdorff, $\theta^{*}(L)$ contains exactly those congruences whose images are also compact Hausdorff. Second, it is not difficult to show that if $L$ is compact Hausdorff then $[\phi] \in \theta^{*}(L)$ implies $\phi$ preserves arbitrary meets and arbitrary joins. The importance of this last statement is twofold:

(i) every compact Hausdorff topological lattice is complete;

(ii) a homomorphism of a complete lattice that does not preserve arbitrary meets and arbitrary joins may produce an image radically different from the domain (cf. [2]).

Finally in all that follows, unless otherwise stated, $L$ will always be a topological lattice, and $I$ will denote the unit interval $[0,1]$ with the usual topology.

1. Let us first state some facts concerning the construction of both congruences and closed congruences.

Throughout this section $X$ is assumed to be a subset of $L \times L$, and $X^{t}$ will denote the transitive closure of $X$.

(1.1) Remark. If $X$ is a $D$-ideal then $X^{t}$ is a $D$-ideal.

Proof. Note that $D \cup\left(D \vee X^{t}\right) \cup\left(D \wedge X^{t}\right) \subseteq D \cup(D \vee X)^{t} \cup(D \wedge X)^{t} \subseteq$ $X^{t}$.

When $X$ is also symmetric (that is, $(x, y) \in X$ implies $(y, x) \in X)$, we get

(1.2) RemarK. If $X$ is a symmetric $D$-ideal then $X^{t}$ is a congruence.

Proof. Since $X$ is symmetric and contains the diagonal, $X^{t}$ is an equivalence relation. By Remark (1.1), $X^{t}$ is a $D$-ideal.

If $X$ satisfies Remark (1.2), then $X^{t}$ is the smallest congruence containing $X$. However, there is no guarantee that $X^{t}$ is topologically closed. Thus, to construct the smallest closed congruence, we adjoin the limit points of $X^{t}$ obtaining $\mathrm{Cl}\left(X^{t}\right)$. Although this set is a symmetric $D$-ideal (as will be shown), it may not be transitive. Furthermore, the number of times topological and transitive closure must be alternately applied to guarantee a closed congruence may depend on the cardinality of $L \times L$. What we, in fact, create by this alternation process is a collection of sets satisfying the following.

(1.3) Definition. For $X \subseteq L \times L$, let $T X=\mathrm{Cl}\left(X^{t}\right)$, and for each ordinal $j$ we define $T^{j} X$ by transfinite induction such that:

(i) $T^{1} X=T X$,

(ii) $T^{j} X=T\left(T^{j-1} X\right)$, if $j$ is not a limit ordinal,

(iii) $T^{j} X=\cup_{i<j} T^{i} X$, if $j$ is a limit ordinal.

It is evident from this definition that:

(a) for any $X, X \subseteq T X$; hence in particular, $T^{j} X \subseteq T^{k} X$ whenever $j<k$;

(b) a symmetric $D$-ideal is a closed congruence iff $T X=X$.

To further clarify the sets produced in Definition (1.3), we present a series of propositions; the first two of which are used to generate the smallest closed congruence containing a given symmetric $D$-ideal.

(1.4) Proposition. If $X$ is symmetric then all $T^{j} X$ are symmetric. If $X$ is a $D$-ideal then all $T^{j} X$ are $D$-ideals. 
Proof. Since both transitive and topological closure preserve symmetry, $T$ must also. A straightforward transfinite induction yields the symmetry of all $T^{j} X$.

For the second assertion, note that since $L$ is a topological lattice, the topological closure of a $D$-ideal is a $D$-ideal. This together with Remark (1.1) implies that $T X$ is a $D$-ideal. By constructing another transfinite induction, all $T^{j} X$ are $D$-ideals.

Note that set-theoretic considerations require the existence of an ordinal $k$ such that $T^{k} X=T^{j} K$ for all $j \geqslant k$, since otherwise $L \times L$ would have arbitrarily high cardinality. Let $X^{*}$ denote $T^{k} X$.

(1.5) Proposition. If $X$ is a symmetric D-ideal then $X^{*}$ is the smallest closed congruence containing $X$.

Proof. By Proposition (1.4) $X^{*}$ is a symmetric $D$-ideal. Thus, since $T\left(X^{*}\right)=$ $T^{k+1} X=T^{k} X=X^{*}, X^{*}$ is a closed congruence. Let $[\phi]$ be a closed congruence; then for any $Y \subseteq[\phi], \quad Y^{t} \subseteq[\phi]$ and $\mathrm{Cl}(Y) \subseteq[\phi]$. Therefore, if $X \subseteq[\phi]$ then $T X \subseteq[\phi]$ and, by transfinite induction, $T^{j} X \subseteq[\phi]$ for all ordinals $j$. Hence, $X^{*} \subseteq[\phi]$.

At this point the reader might question whether such transfinite inductions as in the above propositions are actually necessary to construct a closed congruence. The answer is affirmative and example (3.1) of the Appendix illustrates this fact.

It follows immediately from Definition (1.3) and the definition of *, that * can be considered as a closure operator on the power set of $L \times L$; that is, for $X$, $Y \subseteq L \times L$ we have

(i) $X \subseteq X^{*}$,

(ii) $X^{*}=X^{* *}$, and

(iii) if $X \subseteq Y$ then $X^{*} \subseteq Y^{*}$.

Similarly, Proposition (1.5) implies that the restriction of * to $\theta(L)$ is also a closure operator.

The following two propositions introduce a set of functions necessary to our investigation of the ${ }^{*}$ operation.

(1.6) Proposition. Let $f$ be a continuous function from $L \times L$ into $L \times L$ such that $f\left(X^{t}\right) \subseteq(f X)^{t}$; then $f\left(X^{*}\right) \subseteq\left(f X^{*}\right)$.

Proof. If $f$ is continuous then $f(\mathrm{Cl}(Y)) \subseteq \mathrm{Cl}(f Y)$ for any $Y \subseteq L \times L$. This together with the condition, $f\left(X^{t}\right) \subseteq(f X)^{t}$, yields $f(T X)=f\left(\mathrm{Cl}\left(X^{t}\right)\right) \subseteq \mathrm{Cl}\left(f\left(X^{t}\right)\right)$ $\subseteq \mathrm{Cl}\left((f X)^{t}\right)=T(f X)$. By applying yet another transfinite induction, we obtain $f\left(T^{j} X\right) \subseteq T^{j}(f X)$ for all ordinals $j$. Hence, $f\left(X^{*}\right) \subseteq(f X)^{*}$.

(1.7) Proposition. Let $(u, v) \in L \times L$ and define $f: L \times L \rightarrow L \times L$ by $f(x, y)$ $=(v \wedge(u \vee x), v \wedge(u \vee y))$. Then $f$ satisfies

(a) Proposition (1.6),

(b) $(u \wedge v, v)=f(u, v)$

(c) if $X$ is a $D$-ideal then $f X \subseteq X$,

(d) if $[\phi]$ is a congruence and $(u, v) \in[\phi]$ then $f(L \times L) \subseteq[\phi]$. 
Proof. (a). Since $L$ is a topological lattice, $f$ is clearly continuous. Moreover, the inclusion, $f\left(X^{t}\right) \subseteq(f X)^{t}$, is straightforward.

(b) Clear.

(c) Note that for any $D$-ideal $X, f X=(v, v) \wedge((u, u) \vee X) \subseteq D \wedge(D \vee X) \subseteq$ $X$.

(d) $(u, v) \in[\phi]$ implies $(v \wedge u, v),(v, v \wedge u) \in[\phi]$. Thus, by letting "o" represent transitivity such that $(a, b) \circ(b, c)=(a, c)$, we have $f(x, y)=(v \wedge(u \vee$ $x), v) \circ(v, v \wedge(u \vee y))=[(v \wedge(u \vee x), v \wedge(u \vee x)) \vee(v \wedge u, v)] \circ[(v \wedge(u$ $\vee y), v \wedge(u \vee y)) \vee(v, v \wedge u)] \in(D \vee[\phi]) \circ(D \vee[\phi])=[\phi] \circ[\phi]=[\phi]$

The following lemma provides the fundamental observation in establishing that the ${ }^{*}$ operation preserves finite intersections when restriced to members of $\theta(L)$.

(1.8) Lemma. If $X$ is a symmetric $D$-ideal and $[\phi] \in \theta(L)$ then $X^{*} \cap[\phi] \subseteq$ $(X \cap[\phi])^{*}=$ smallest closed congruence containing $X \cap[\phi]$.

Proof. Let $(u, v) \in X^{*} \cap[\phi]$. Using the same notation as in Proposition (1.6) and (1.7), recall that $(u \wedge v, v)=f(u, v)$ which is certainly a member of $f\left(X^{*}\right)$. By applying Proposition (1.7a) we obtain $f\left(X^{*}\right) \subseteq(f X)^{*}$. Now Proposition (1.7)(c) and (d) implies $f X \subseteq X \cap[\phi]$, and the monotonicity of ${ }^{*}$ gives $(f X)^{*} \subseteq(X \cap[\phi])^{*}$. Together these inclusions imply that $(u \wedge v, v) \in(X \cap[\phi])^{*}$. A symmetric argument shows that $(u \wedge v, u) \in(X \cap[\phi])^{*}$. Thus, by relying on the symmetry and transitivity of $(X \cap[\phi])^{*}$ we obtain $(u, v) \in(X \cap[\phi])^{*}$.

Finally, note that $X \cap[\phi]$ is a symmetric $D$-ideal; so Proposition (1.5) justifies our last assertion.

To expedite the remainder of our investigation, we define a map, ${ }^{*}$, from $\theta(L)$ onto $\theta^{*}(L)$ such that ${ }^{*}([\phi])=[\phi]^{*}$. That is, the map ${ }^{*}$ is the restriction of the canonical map associated with the operation * such that the domain and range of the map are restricted to $\theta(L)$ and $\theta^{*}(L)$ respectively. Of course the * map may also be viewed as a closure operator on $\theta(L)$.

(1.9) ThEOREM. The map ${ }^{*}$ from $\theta(L)$ onto $\theta^{*}(L)$ preserves finite meets (i.e. intersections) and arbitrary joins.

Proof. Let $[\phi],[\Psi] \in \theta(L)$. Then ${ }^{*}([\phi] \cap[\Psi]) \subseteq{ }^{*}([\phi]) \cap{ }^{*}([\Psi])$ is derived from the monotonicity of the ${ }^{*}$ operation. For the reverse containment, we have

$$
\begin{aligned}
*([\phi]) \cap{ }^{*}([\Psi]) & =[\phi]^{*} \cap[\Psi]^{*} \subseteq\left([\phi] \cap[\Psi]^{*}\right)^{*} \quad \text { by Lemma }(1.8) \\
& \subseteq([\phi] \cap[\Psi])^{* *} \quad \text { by Lemma }(1.8) \text { and the monotonicity of * } \\
& \subseteq([\phi] \cap[\Psi])^{*} \quad \text { by the idempotency of * } \\
& ={ }^{*}([\phi] \cap[\Psi]) .
\end{aligned}
$$

Thus, the ${ }^{*}$ map preserves finite meets.

The argument for arbitrary joins is not difficult and can be shown directly, or may be based on the fact that a closure operator on any lattice preserves arbitrary joins whenever they exist. 
There are quite simple examples which show that the ${ }^{*}$ map does not preserve arbitrary meets. Example (3.2) of the Appendix illustrates this for the ${ }^{*}$ map from $\theta(I)$ onto $\theta^{*}(I)$.

(1.10) Remark. If $g$ is a function from a complete Brouwerian lattice $A$ onto a complete lattice $B$ such that $g$ preserves finite meets and arbitrary joins then $B$ is a complete Brouwerian lattice.

Proof. $g$ preserves the defining infinitary equation for Brouwerian lattices.

(1.11) TheOREM. $\theta^{*}(L)$ is a complete Brouwerian lattice.

Proof. $\theta(L)$ is a complete Brouwerian lattice. Hence the theorem follows from Theorem (1.9) via Remark (1.10).

(1.12) Corollary. $\theta^{*}(L)$ is a distributive latice.

Proof. Any Brouwerian lattice is distributive (cf. [1, pp. 45-46]).

Since $\theta^{*}(L)$ satisfies one of the infinite distributive laws, a natural question is: does it satisfy the other? That is, for $\left[\phi_{\alpha}\right],[\Psi] \in \theta^{*}(L), \alpha \in \mathcal{Q}$, is $\left(\cap_{\alpha \in \mathcal{Q}}\left[\phi_{\alpha}\right]\right) \forall[\Psi]$ $=\cap{ }_{\alpha \in \mathcal{Q}}\left(\left[\phi_{\alpha}\right] \forall[\Psi]\right)$ also true? The answer is negative, and in fact even more can be said. A complete lattice $M$ is lower continuous if whenever there exists a monotone decreasing chain $\left\{d_{\alpha} \mid \alpha \in \mathbb{Q}\right\}$ in $M$ then

$$
\left(\bigwedge_{\alpha \in \mathbb{Q}} d_{\alpha}\right) \vee x=\bigwedge_{\alpha \in \mathbb{Q}}\left(d_{\alpha} \vee x\right)
$$

for any $x \in M$. In Example (3.3) of the Appendix we show that $\theta^{*}(I)$ is not lower continuous.

To obtain the final results of this section a few preliminary remarks are in order.

If $a$ and $b$ are elements of the lattice $M$, then the maximal element of $\{x \in M \mid a$ $\wedge x \leqslant b\}$ (when it exists) is called the relative pseudo-complement of $a$ in $b$, and is denoted $b: a$.

A Brouwerian lattice $M$ (not necessarily complete) is defined to be a lattice such that any pair of elements in $M$ have a relative pseudo-complement (cf. [1, pp. 45-46, 128]).

If $M$ is a lattice with a least element, 0 , and $a \in M$, then the element 0: $a$ (when it exists) is referred to as the pseudo-complement of $a$.

In a complete Brouwerian lattice every element is pseudo-complemented, and it is well known that the set of pseudo-complements with the inherited order form a complete Boolean algebra in which the meet is the same as in the original lattice (cf. [1, p. 129]).

The following proposition provides sufficient information to relate the * map and the relative pseudo-complements of $\theta(L)$ and $\theta^{*}(L)$.

(1.13) Proposition. Let $M$ be a lattice and $\mathcal{C}$ a closure operator on $M$ that preserves finite meets. If $a, b \in M, C b=b$, and $(b: a)$ exists then $(b: a)=(b$ : $C a)=C(b: a)=$ relative pseudo-complement of $C a$ in $b$ with respect to the lattice eM. 
Proof. Let " $\ell$ " denote the meet in $C M$.

Claim. For any $c, d \in M, e_{c} \wedge e_{d}=\mathcal{C}(c \wedge d)=e_{c} \ell e_{d}=\mathcal{C}\left(C_{c} \wedge d\right)$.

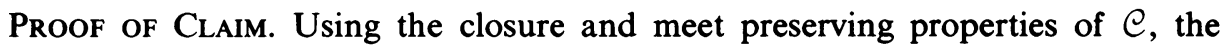
following chain of inequalities proves our claim:

$$
\begin{aligned}
& e_{c} \wedge e_{d} \geqslant e(c \wedge d)=e_{c} \ell e_{d}=e\left(e_{c}\right) \ell e_{d}=e\left(e_{c} \wedge d\right) \\
& =e\left(e_{c}\right) \ell \mathcal{C}\left(e_{d}\right)=e\left(e_{c} \wedge e_{d}\right) \geqslant e_{c} \wedge e_{d}
\end{aligned}
$$

To show $(b: a)=(b: \bigodot a)$, we have

$$
\begin{aligned}
a \wedge(b: C a) & \leqslant C(a \wedge(b: C a))=\mathcal{C}(C a \wedge(b: C a)) \quad \text { (by the Claim) } \\
& \leqslant b, \text { since } C a \wedge(b: C a) \leqslant b \text { and } C b=b .
\end{aligned}
$$

Thus, $(b: C a) \leqslant(b: a)$ by the maximality of $(b: a)$. For the reverse inequality,

$$
\begin{aligned}
C a \wedge(b: a) & \leqslant C(C a \wedge(b: a))=\mathcal{C}(a \wedge(b: a)) \quad \text { (by the Claim) } \\
& \leqslant b .
\end{aligned}
$$

So, $(b: a)=(b: C a)$.

For the second equality we have $C a \wedge C(b: a)=C(a \wedge(b: a))$ (by the Claim) $\leqslant b$. So, $\mathcal{C}(b: a) \leqslant(b: \mathcal{C} a)=(b: a) \leqslant \mathcal{C}(b: a)$. Therefore equality holds.

To show that $(b: a)$ is the relative pseudo-complement of $C a$ in $b$ with respect to $\mathcal{C} M$, note first that $(b: a)=\mathcal{C}(b: a) \in \mathcal{C}$. Now if $x \in \mathcal{C} M$ (i.e., $x=\mathcal{C}_{x}$ ) and $C a \ell x \leqslant b$, then $C a \wedge x \leqslant b$ by the Claim. Thus, $x \leqslant(b: C a)=(b: a)$.

(1.14) RemarK. Proposition (1.13) implies that the relative pseudo-complements in $b$ with respect to the lattices $M$ and $C M$ are the same. Therefore, upon substituting $\theta(L)$ for $M$, and * for $C$ in Proposition (1.13) we see that for $[\phi] \in \theta^{*}(L)$ the set of relative pseudo-complements in $[\phi]$ with respect to either $\theta(L)$ or $\theta^{*}(L)$ is the same. In particular, when considering pseudo-complements we obtain the following result.

(1.15) THEOREM. $L$ is Hausdorff iff the pseudo-complements of $\theta(L)$ and $\theta^{*}(L)$ are identical.

Proof. If $L$ is Hausdorff then $D$ is a closed congruence. Substituting $D$ for $[\phi]$ in Remark (1.14) implies that $\theta(L)$ and $\theta^{*}(L)$ have the same pseudo-complements.

Conversely, since $D$ is the pseudo-complement of $L \times L$ in $\theta(L)$, we get $D \in \theta^{*}(L)$ which implies $L$ is Hausdorff.

(1.16) Corollary. L is compact Hausdorff iff the pseudo-complements of $\theta(L)$ have compact Hausdorff images.

Proof. $L$ is compact Hausdorff iff the image of each closed congruence is compact Hausdorff. This together with Theorem (1.15) gives the result in the forward direction. The converse is identical to that of Theorem (1.15).

2. In discussing the topological nature of lattices of closed congruences, the following definitions and remarks will be useful.

A topological semilattice is a semilattice endowed with a topology such that the semilattice operation (whether meet, or join) is continuous. If $S$ is a topological semilattice such that the meet (join) is continuous, then we define a semilattice 
congruence $[\phi]$ as an equivalence relation on $S$ such that $D \cup(D \wedge[\phi]) \subseteq[\phi]$ (or, $D \cup(D \vee[\phi]) \subseteq[\phi]$ in the join case). Of course, the remarks in the preliminaries are also applicable in the context of a topological semilattice $S$, its semilattice congruences $\theta(S)$, and its closed semilattice congruences $\theta^{*}(S)$.

Also, note that any semilattice congruence on $I$ is a lattice congruence. Thus, $\theta^{*}(I)$ denotes both the closed semilattice congruences and the closed lattice congruences.

The key to our result lies in the following theorem where we employ an argument similar to one used by E. E. Floyd in [3].

\section{(2.1) ThEOREM. There does not exist a compatible Hausdorff topology on $\theta^{*}(I)$.}

Proof. Suppose $\mathcal{T}$ is a compatible topology on $\theta^{*}(I)$. Let $\left\{U_{i}\right\}_{i=1}^{\infty}$ be an open base for the topology on $I$ such that $\mathrm{Cl}\left(U_{i}\right) \cap \mathrm{Cl}\left(U_{i-1}\right)=\varnothing$. Let $\left[\psi_{i}\right]=\{(x, y) \mid x, y$ $\left.\in \mathrm{Cl}\left(U_{i}\right)\right\} \cup D$; thus, $\left[\psi_{i}\right] \in \theta^{*}(I)$ and $\left[\psi_{i}\right] \cap\left[\psi_{i-1}\right]=D$. It is not difficult to show that for each positive integer $i$ there is a sequence $\left\{\left[\phi_{i j}\right]\right\}_{j=1}^{\infty} \subseteq \theta^{*}(I)$ such that the terms of the sequences have the following relationships.

(i) $\left[\psi_{i}\right] \subseteq\left[\phi_{i j}\right] \neq I \times I$,

(ii) $\left[\psi_{i-1}\right] \nsubseteq\left[\phi_{i j}\right]$,

(iii) $\left[\phi_{i j}\right] \subseteq\left[\phi_{i(j+1)}\right]$,

(iv) $\forall_{j=1}^{\infty}\left[\phi_{i j}\right]=I \times I$.

Let $\theta$ be any neighborhood of the congruence $I \times I$ in $\mathcal{T}$. By the compatibility of $\mathcal{T}$, the sequence $\left\{\left[\phi_{1 j}\right]\right\}_{j=1}^{\infty}$ converges to $I \times I$ in $\mathcal{T}$. Hence, there exists $\left[\phi_{1 j}\right] \in \mathcal{O}$ for some $j$. Let $\left[\rho_{1}\right]=\left[\phi_{1 j}\right]$ for some such $j$. Now $\left[\rho_{1}\right]=(I \times I) \cap\left[\rho_{1}\right]=\left(\forall_{j=1}^{\infty}\left[\phi_{2 j}\right]\right)$ $\cap\left[\rho_{1}\right]=\forall_{j=1}^{\infty}\left(\left[\phi_{2 j}\right] \cap\left[\rho_{1}\right]\right)$ by Theorem (1.11). Since the sequence $\left\{\left[\phi_{2 j}\right] \cap\left[\rho_{1}\right]\right\}_{j=1}^{\infty}$ is monotone increasing, the compatibility forces convergence to $\left[\rho_{1}\right]$. Therefore, this sequence is also eventually in $\mathcal{\theta}$. Define $\left[\rho_{2}\right]=\left[\phi_{2 j}\right] \cap\left[\rho_{1}\right]$ where $\left[\phi_{2 j}\right] \cap\left[\rho_{1}\right] \in \mathcal{O}$. Note that $\left[\rho_{2}\right]$ does not contain $U_{1}$ in any equivalence class.

Similarly, $\left[\rho_{2}\right]=(I \times I) \cap\left[\rho_{2}\right]=\left(\forall_{j=1}^{\infty}\left[\phi_{3 j}\right]\right) \cap\left[\rho_{2}\right]=\forall_{j=1}^{\infty}\left(\left[\phi_{3 j}\right] \cap\left[\rho_{2}\right]\right)$ and, by compatibility, $\left\{\left[\phi_{3 j}\right] \cap\left[\rho_{2}\right]\right\}_{j=1}^{\infty}$ converges to $\left[\rho_{2}\right]$. Again, pick $j$ such that $\left[\phi_{3 j}\right] \cap\left[\rho_{2}\right]$ $\in \mathcal{O}$ and call this element $\left[\rho_{3}\right]$. Note that $\left[\rho_{3}\right]$ does not contain $U_{1}$ or $U_{2}$ in any equivalence class.

Continuing, we obtain the sequence $\left\{\left[\rho_{i}\right]\right\}_{i=1}^{\infty}$ such that $\left[\rho_{i+1}\right] \subseteq\left[\rho_{i}\right]$.

By the compatibility of $\mathcal{T},\left\{\left[\rho_{i}\right]\right\}_{i=1}^{\infty}$ must converge to $\bigcap_{i=1}^{\infty}\left[\rho_{i}\right]$. Also, since $\left\{\left[\rho_{i}\right]\right\}_{i=1}^{\infty} \subseteq \vartheta$ we have $\cap_{i=1}^{\infty}\left[\rho_{i}\right] \in \mathrm{Cl}(\theta)$. However, $\cap_{i=1}^{\infty}\left[\rho_{i}\right]$ contains no $U_{i}$ in any equivalence class. Therefore $\bigcap_{i=1}^{\infty}\left[\rho_{i}\right]=D$. This means that every neighborhood $\theta$ of the element $I \times I$ in $\mathcal{T}$ contains the congruence $D$ in its closure $\mathrm{Cl}(\theta)$. So $\mathcal{T}$ is not Hausdorff.

(2.2) Corollary. If $L$ is a topological (semi) lattice with the unit interval as a (semi) lattice homomorphic image, then the lattice of closed (semi) lattice congruences has no compatible Hausdorff topology.

Proof. The following proof suffices for both cases. Suppose $\mathcal{T}$ is a compatible Hausdorff topology for $\theta^{*}(L)$. Let $\phi$ be the homomorphism from $L$ onto $\phi(L)=I$. Thus, $[\phi] \in \theta^{*}(L)$. If $[\rho] \in \theta^{*}(L)$ such that $[\phi] \subseteq[\rho]$ then there exists a homomorphism $\rho_{\phi}$ from $I=\phi(L)$ onto $\rho(L)$ such that the following diagram commutes. 


$$
\begin{aligned}
& \phi(L)=I \\
& \phi \nearrow \quad \searrow \rho_{\phi} \\
& L \quad \vec{\rho} \quad \rho(L)
\end{aligned}
$$

Let $(\uparrow[\phi])=\left\{[\rho] \in \theta^{*}(L) \mid[\phi] \subseteq[\rho]\right\}$. It is easy to show that the map $g$ from $(\uparrow[\phi])$ into $\theta^{*}(I)$ such that $g([\rho])=\left[\rho_{\phi}\right]$ is a complete lattice isomorphism. Moreover, the restriction of $\mathcal{T}$ to $(\uparrow[\phi])$ is a compatible Hausdorff topology. Therefore by making $U$ open in $\theta^{*}(I)$ iff $g^{-1}(U)$ is open, we obtain a compatible Hausdorff topology for $\theta^{*}(i)$, a contradiction to Theorem (2.1).

\section{Appendix.}

(3.1) ExAmple. The transfinite inductions in $\$ 1$ are necessary. For $S \subset L \times L$, let $[S]$ be the smallest congruence containing $S$. Let $[a, b], a<b$, be a line segment from the unit interval. Let $\left[\psi_{1}\right]$ denote the following congruence on $[a, b]:\{(x, y) \mid b$ $-(b-a) / 2^{n} \leqslant x, y<b-(b-a) / 2^{n+1}$ for some $\left.n=0,1,2, \ldots\right\}$. Pictorially, $\left[\psi_{1}\right]$ may be represented as

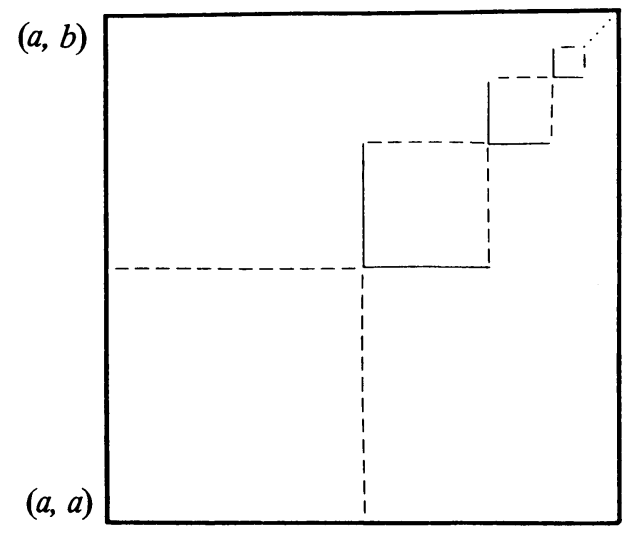

$(b, b)$

$(b, a)$

It is not difficult to show that $\left[\mathrm{Cl}\left[\psi_{1}\right]\right]$ has the following diagram.

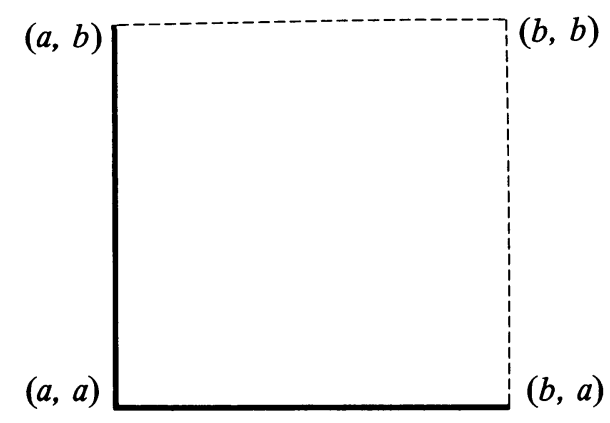

Thus, $\mathrm{Cl}\left[\mathrm{Cl}\left[\psi_{1}\right]\right]=[a, b] \times[a, b]$ is the closed congruence generated by $\left[\psi_{1}\right]$. 
Let

$$
\begin{aligned}
{\left[\psi_{2}\right]=\{(x, y) \mid b} & -\left((b-a) / 2^{n+1}\right)\left(1+1 / 2^{m}\right) \leqslant x, y \\
& \left.<b-\left((b-a) / 2^{n+1}\right)\left(1+1 / 2^{m+1}\right) \text { for } n, m=0,1,2,3, \ldots\right\} .
\end{aligned}
$$

Note that $\left[\psi_{2}\right]$ is also a congruence on $[a, b]$, and has the following diagram. Moreover, it is not difficult to show that $\left[\mathrm{Cl}\left[\psi_{2}\right]\right]=\left[\psi_{1}\right]$. Hence to obtain the closed congruence generated by $\left[\psi_{2}\right]$, we must alternate the closure and congruence generating operators such that the closure operator is applied three times.

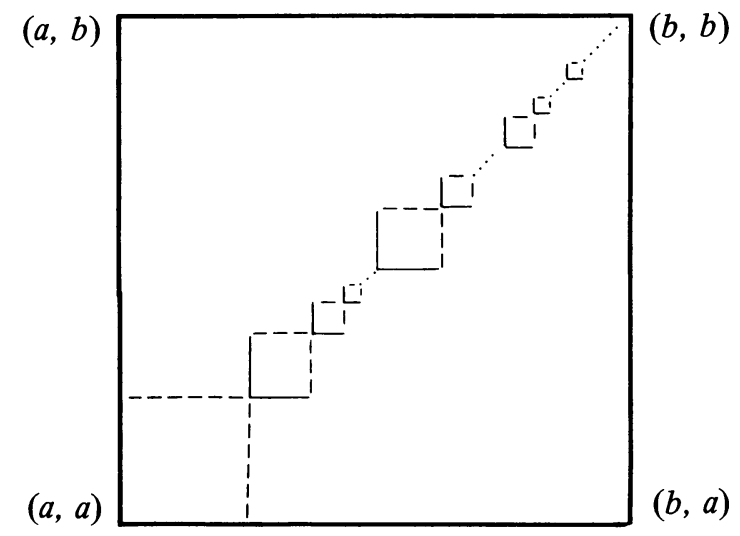

Similarly, we can define a congruence $\left[\psi_{3}\right]$ such that $\left[\mathrm{Cl}\left[\psi_{3}\right]\right]=\left[\psi_{2}\right]$. Continuing, a congruence $\left[\psi_{n}\right]$ can be defined for each positive integer such that $\left[\mathrm{Cl}\left[\psi_{n}\right]\right]=$ $\left[\psi_{n-1}\right]$ whenever $n>2$.

Starting with the diagonal $D$, we construct our example as follows.

First replace $\{(x, x) \mid 1 / 3 \leqslant x \leqslant 2 / 3\}$ with the congruence $\left[\psi_{1}\right]$ on $[1 / 3,2 / 3]$ to get the diagram

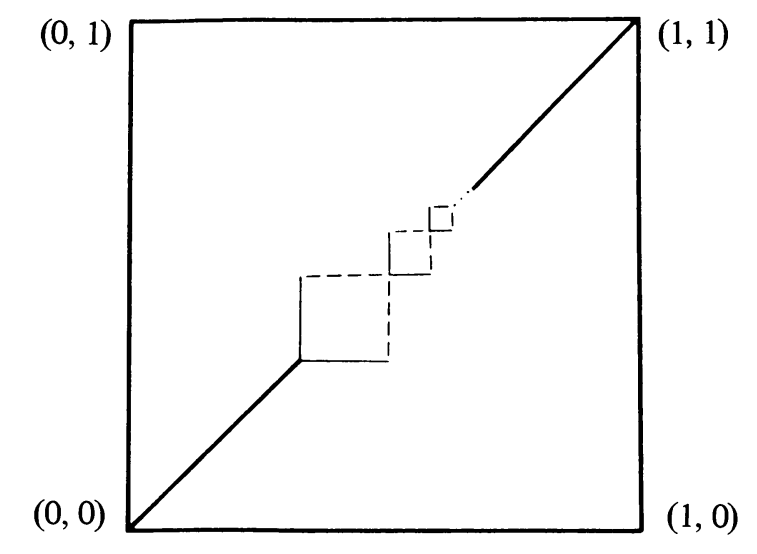

Next, replace $\{(x, x) \mid 1 / 9 \leqslant x \leqslant 2 / 9\}$ and $\{(x, x) \mid 7 / 9 \leqslant x \leqslant 8 / 9\}$ by $\left[\psi_{2}\right]$ on the intervals $[1 / 9,2 / 9]$ and $[7 / 9,8 / 9]$, respectively.

For the $n$th step replace the middle third of each untouched segment of the diagonal with $\left[\psi_{n}\right]$. Let $[S]$ denote the resulting set. Thus, $[S]$ can be represented as follows. 


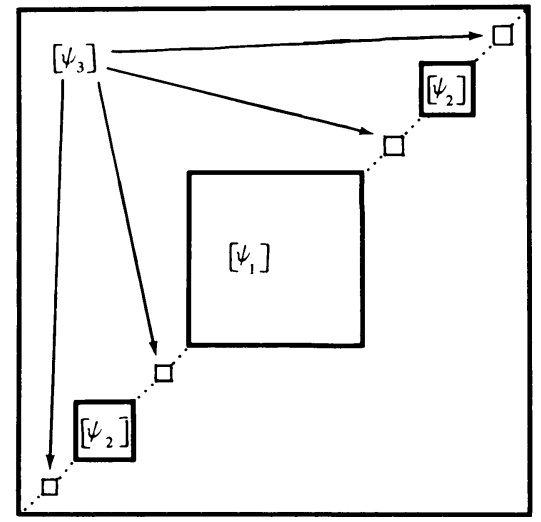

It is not difficult to show that $[S]$ is a congruence, and moreover, no finite number of applications of closure and congruence generation will suffice to produce the closed congruence associated with $[S]$.

(3.2) Example. The map ${ }^{*}$ from $\theta(I)$ onto $\theta^{*}(I)$ does not preserve arbitrary meets.

Proof. Let $\left[\rho_{n}\right]=\left\{(r, s) \mid k / 2^{n}<s, r<(k+1) / 2^{n}, k=0,1, \ldots, 2^{n}-1\right\} \cup D$, for each positive number $n$. It is easy to show $\left[\rho_{n}\right] \in \theta(L)$ for all $n$. Now $\cap_{n=1}^{\infty}\left[\rho_{n}\right]=D$, so ${ }^{*}\left(\cap_{n=1}^{\infty}\left[\rho_{n}\right]\right)=D$. However, $\left[\rho_{n}\right]^{*}=I \times I$ for all $n$, so $\cap_{n=1}^{\infty}\left[\rho_{n}\right]^{*}=I \times I$.

(3.3) EXAMPLE. $\theta^{*}(I)$ is not lower continuous.

Proof. For each positive integer $n$, let $\left[\rho_{n}\right]=\left\{(r, s) \in I \times I \mid k / 3^{n} \leqslant r, s<\right.$ $\left.(k+1) / 3^{n}, k=0,2,4,6, \ldots, 3^{n}-1\right\} \cup D$. It is not difficult to see that $\left[\rho_{n}\right]$ is a closed congruence for each $n$. Let $\left[\psi_{i}\right]=\bigcap_{n=1}^{i}\left[\rho_{n}\right]$, so $\left[\psi_{i}\right] \in \theta^{*}(L)$. Let $[\phi]=$ $\{(r, s) \in I \times I \mid r, s$ are in the same component of $\overline{I-C}$ where $C$ is the Cantor set $\} \cup D$. It is also easy to see that $[\phi] \in \theta^{*}(L)$. Now $\left[\psi_{n}\right] \forall[\phi]=I \times I$ for all $n$. Hence $\cap_{n=1}^{\infty}\left(\left[\psi_{n}\right] \forall[\phi]\right)=I \times I$. But $\cap_{n=1}^{\infty}\left[\psi_{n}\right]=D$, so $\left(\cap_{n=1}^{\infty}\left[\psi_{n}\right]\right) \forall[\phi]=[\phi]$. Therefore $\left(\bigcap_{n=1}^{\infty}\left[\psi_{n}\right]\right) \forall[\phi] \neq \bigcap_{n=1}^{\infty}\left(\left[\psi_{n}\right] \forall[\phi]\right)$.

\section{REFERENCES}

1. G. Birkhoff, Lattice theory, Amer. Math. Soc. Colloq. Publ., vol. 25, Amer. Math. Soc., Providence, R. I., 1967.

2. P. Crawley and R. P. Dilworth, Algebraic theory of lattices, Prentice-Hall, Englewood Cliffs, N. J., 1973.

3. E. E. Floyd, Boolean algebras with pathological order topologies, Pacific J. Math. 5 (1955), 687-689.

4. K. H. Hofmann and M. W. Mislove, The lattice of kernel operators and topological algebra, Math. Z. 154 (1977), 175-188.

5. K. H. Hofmann, M. Mislove and A. Stralka, The Pontryagin duality of compact 0-dimensional semilattices and its applications, Lecture Notes in Math., vol. 396, Springer-Verlag, Berlin and New York, 1974.

6. A. Stralka, The congruence extension property for compact topological lattices, Pacific J. Math. 38 (1971), 795-802.

Department of Computer Science, University of Colorado, Boulder, Colorado 80309 